\title{
FONTES DE INFORMAÇÕES SOBRE INTERAÇÕES MEDICAMENTOSAS: HÁ CONCORDÂNCIA ENTRE ELAS?
}

\author{
Camilo Molino Guidoni $\mathrm{MSc}^{{ }^{*}}$ \\ André de Oliveira Baldoni MSc ${ }^{1}$ \\ Paulo Roque Obreli-Neto $\mathrm{MSc}^{2}$ \\ Leonardo Régis Leira Pereira $\mathrm{PhD}^{1}$
}

\begin{abstract}
${ }^{1}$ Centro de Pesquisa em Assistência Farmacêutica e Farmácia Clínica (CPAFF), Departamento de Ciências Farmacêuticas, Faculdade de Ciências Farmacêuticas de Ribeirão Preto, Universidade de São Paulo, Brasil .

${ }^{2}$ Departamento de Farmacologia e Terapêutica, Universidade Estadual de Maringá, Brasil.
\end{abstract}

*Correspondência: MSc Camilo Molino Guidoni, Departamento de Ciências Farmacêuticas, Faculdade de Ciências Farmacêuticas de Ribeirão Preto, Universidade de São Paulo, Brasil, Avenida do Café, s/nº - Campus Universitário da USP - Ribeirão Preto - SP - 14040-903 - Brasil. Telefone: 55 (16) 3602-4236. E-mail: camiloguidoni@ yahoo.com.br

RESUMO: Introdução. A informação segura e correta sobre os medicamentos constitui uma etapa essencial para promoção do uso racional dos medicamentos, principalmente de medicamentos com elevado número de interações medicamentosas como a varfarina. Objetivo. Correlacionar cinco fontes de informações sobre interações medicamentosas utilizando, como instrumento de comparação, as potenciais interações relacionados à varfarina. Casuística e Métodos. Trata-se de estudo descritivo. Foram selecionadas cinco fontes de informações sobre interações medicamentosas: Micromedex®, Guia de Interações Medicamentosas da Organização Mundial de Saúde: "Model Formulary, 2008", "Guia Zanini-Oga de Interações medicamentosas", PR Vade Mécum ${ }^{\circledR}$, Bula do medicamento de referência da varfarina. As variáveis estudadas foram: número de interações, presença de interação fármaco-alimento e/ou fármaco-álcool, classificação da gravidade das interações, número de interações consideradas graves, forma de apresentação da interação (fármaco específico ou classe farmacológica), e identificação e classificação de acordo com a Anatomical Therapeutic Chemical (ATC) dos fármacos com potencial interação com a varfarina. Resultados e Discussão. O número de interações encontradas variou de 46 a 326, uma fonte não considerou interação com alimento e/ou álcool, apenas duas fontes classificaram as interações de acordo com a gravidade, nenhuma interação envolvendo o mesmo fármaco foi detectada simultaneamente nas cinco fontes de informação. Observou-se maior prevalência de fármacos com potencial interação com varfarina que atuam no sistema nervoso. Conclusão. As fontes de informações apresentaram perfis heterogêneos relacionados às interações envolvendo a varfarina, destacando-se a discrepância entre a quantidade, a classificação e forma de apresentação das interações medicamentosas.

Palavras-chave: Interações medicamentosas. Fontes de informações. Fontes bibliográficas. Micromedex. Varfarina.

ABSTRACT: Introduction. The reliable and correct information about drugs is an essential step to promote the rational use of drugs, particularly drugs with a high number of interactions such as warfarin. Objective. To correlate five information sources about drug interactions using warfarin as an instrument of comparison. Casuistic and Methods. It is a descriptive study. It was selected five information sources about drug interactions: Micromedex®, World Health Organization Model Formulary 2008, "Guia Zanini-Oga de Interações medicamentosas”, PR Vade Mécum®, Package insert of brand warfarin. The variables studied were: number of interactions, the presence of drug-food or drug-alcohol interactions or both, classification of the severity of interactions, number of interactions considered major, manner of interaction presentation (specific drug or pharmacologic group), and identification and classification according to the Anatomical Therapeutic Chemical (ATC) of drugs with potential interaction with warfarin. Results and Discussion. The number of interactions provided by the information sources ranged from 46 to 326, one source did not consider interaction with food or alcohol or both, only two sources classified according to severity of interactions, no drug was present simultaneously in all information sources. Nervous System drugs were the most prevalent drugs with potential interaction with warfarin. Conclusion. The information sources presented heterogeneous profiles in relation to 
interactions involving warfarin, emphasizing the discrepancy among amount, classification and presentation of drug interactions.

Keywords: Drug Interaction. Information Sources. Bibliographic Sources. Micromedex. Warfarin.

\section{INTRODUÇÃO}

A informação sobre medicamentos constitui parte essencial de uma política nacional de medicamentos, e para garantia da qualidade e eficiência dessas informações, estas devem ser "seguras, imparciais, independentes, atualizadas, objetivas, e imune a pressões políticas e econômicas", além disso, "deve ser fornecida com agilidade, em tempo hábil para sua utilização de forma individualizada" (Marin, 2003). Neste contexto, as interações medicamentosas (IM) fazem parte do conjunto de informações necessárias para a promoção do uso racional e correto dos medicamentos.

Diante disto, é importante considerar que os profissionais de saúde devem conhecer desde as fontes de informações disponíveis até os efeitos clínicos das IM. Este conhecimento pode auxiliar no cuidado do paciente e evitar problemas farmacoterapêuticos, pois as IM podem gerar conseqüências clínicas, humanísticas e econômicas para o paciente e para o sistema de saúde, podendo favorecer o aparecimento das reações adversas (CruciolSouza JM, Thomson, 2006), além de comprometer o quadro clínico e elevar a taxa de hospitalização (Wasserfallen et al, 2001; Patel et al, 2007; Kongkaew C, Noyce PR, Ashcroft
DM., 2008), prejudicando a qualidade de vida do paciente (Baiardini et al, 2004).

Sendo assim, devido à diversidade e divergência quanto às informações referentes às IM e os riscos que estas trazem para o cuidado do paciente (Cruciol-Souza JM, Thomson, 2006), o profissional de saúde deve conhecer e analisar criticamente as diferentes fontes de informações disponíveis na literatura. Dessa forma, o presente estudo tem como objetivo correlacionar cinco fontes de informações sobre IM utilizando, como instrumento de comparação as potenciais interações relacionados a varfarina.

\section{CASUÍSTICA E MÉTODOS}

Realizou-se um estudo descritivo para analisar a concordância entre cinco fontes de informações sobre IM. A escolha destas foi baseada na acessibilidade, abrangência nacional e internacional, idioma de publicação, e perfil dos usuários, de forma que se tentou abranger fontes utilizadas por profissionais que atuem na área de ensino, pesquisa e clínica.

Baseando-se nestas características e no consenso entre os autores, foram selecionadas as seguintes fontes de informações: Micromedex® (2011) disponível online (acesso 
restrito) (Micromedex, 2011); Guia de Interações Medicamentosas da Organização Mundial de Saúde: "Model Formulary, 2008", disponível online (acesso livre) (OMS, 2008); Livro acadêmico "Guia Zanini-Oga de Interações medicamentosas", disponível impresso (acesso restrito) (Seizi, Basile, Carvalho, 2002); PR Vade Mécum® disponível online (acesso livre) (PR Vade Mecum, 2011); Bula do medicamento de referência da varfarina disponível online no site da Agência Nacional de Vigilância Sanitária (acesso livre) (ANVISA, 2011).

O fármaco varfarina foi escolhido para a análise da concordância entre as fontes de informações devido ao elevado número de potenciais IM descritas na literatura. Além disso, tem sido o pilar da terapêutica anticoagulante oral há aproximadamente 50 anos, está entre os 10 medicamentos mais comumente envolvidos com reações adversas, apresenta estreita janela terapêutica, exibe enorme variabilidade em termos de doseresposta e elevada complexidade de regime posológico (Winterstein et al., 2002; Ansel et al., 2004; Cruciol-Souza, Thomson, 2006; Rane, Lindh, 2010).

Para comparação entre as fontes de informações foram utilizadas as seguintes variáveis: número de interações presente em cada fonte de informação, presença de interação fármaco-alimento e/ou fármaco-álcool, classificação da gravidade das interações, número de interações consideradas graves, forma de apresentação da IM (fármaco específico ou classe farmacológica), e identificação dos fármacos com potencial interação com a varfarina.

Os fármacos identificados nas cinco fontes de informações como medicamentos que interagem com a varfarina foram classificados de acordo com o grupo anatômico principal do primeiro nível de classificação da Anatomical Therapeutic Chemical (ATC) Classification System (ATC/OMS) (OMS, 2011). Vale salientar que um mesmo fármaco pode apresentar mais de um grupo anatômico, sendo inserido em mais de uma categoria da ATC/OMS.

\section{RESULTADOS}

$\mathrm{Na}$ tabela 1 e 2 observa-se a comparação entre as fontes de informações analisadas.

Na tabela 2 estão agrupados os fármacos com potencial IM com varfarina que foram comuns a duas ou mais fontes de informações, destacando-se que nenhum fármaco foi comum as todas as fontes analisadas. 
Tabela 1 - Comparação entre as cinco fontes de informações consultadas para análise de interação com a varfarina.

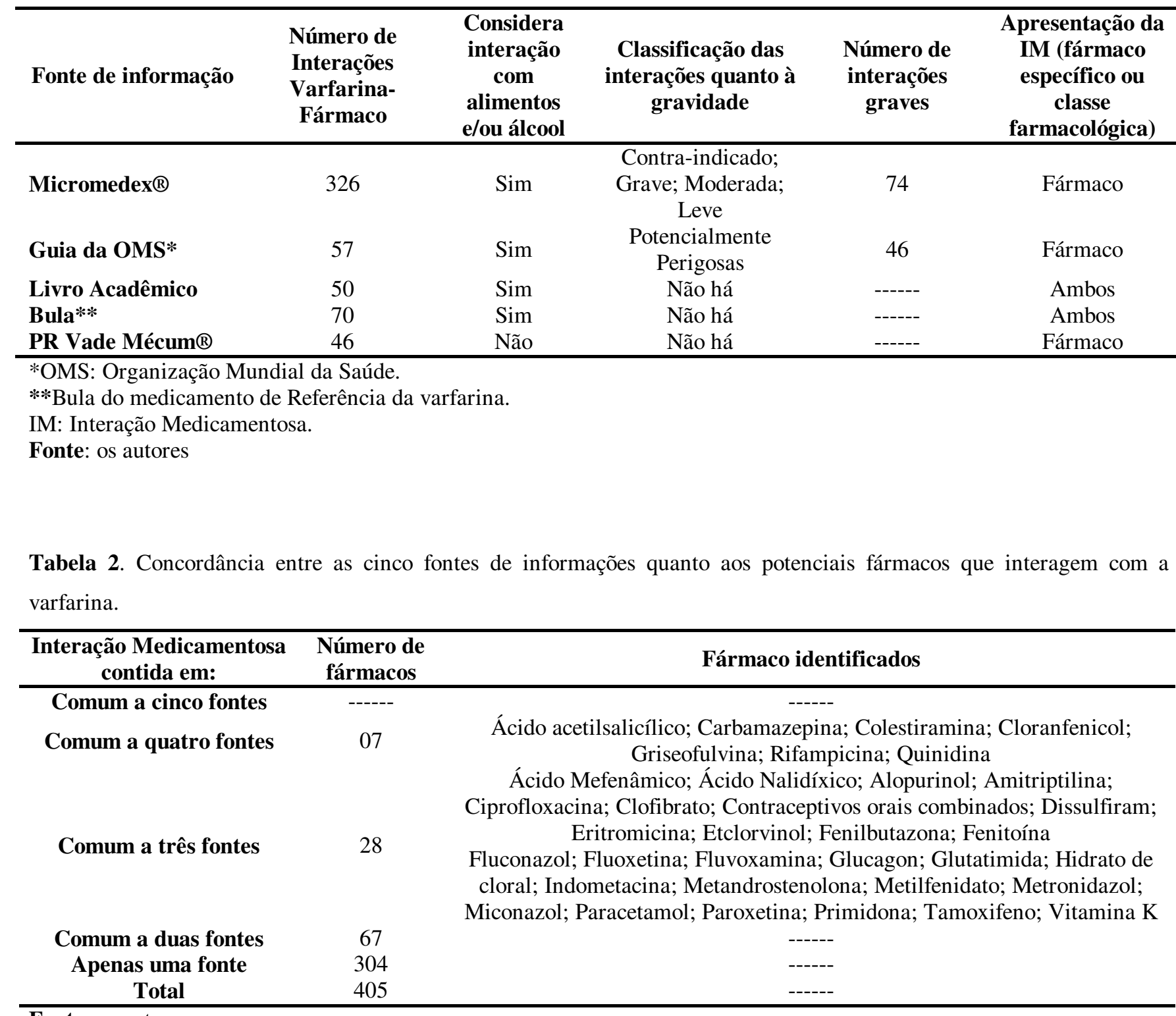

Fonte: os autores

Aproximadamente $50 \%$ dos fármacos

que apresentam potencial interação com a

varfarina estão concentrados em apenas três grupos anatômicos da classificação da

ATC/OMS (Tabela 3). 
Tabela 3. Classificação dos fármacos com potencial interação com a varfarina de acordo com o grupo anatômico principal da Anatomical Therapeutic Chemical Classification System da Organização Mundial da Saúde (ATC/OMS).

\begin{tabular}{ccc}
\hline Código ATC/OMS & Grupo Anatômico Principal & Fármaco (\%) \\
\hline N & Sistema nervoso & 18,0 \\
J & Anti-infecciosos gerais para uso sistêmico & 17,3 \\
M & Sistema músculo-esquelético & 13,8 \\
A & Aparelho digestivo e metabolismo & 10,4 \\
C & Sistema cardiovascular & 10,4 \\
B & Sangue e órgãos hematopoiéticos & 9,6 \\
L & Agentes antineoplásicos e imunomoduladores & 8,9 \\
G & Aparelho geniturinário e hormônios sexuais & 5,4 \\
H & Preparações hormonais sistêmicas, excluindo hormônios sexuais e insulinas & 4,4 \\
S & Órgãos sensoriais & 4,2 \\
D & Dermatológicos & 3,2 \\
P & Produtos antiparasitários, inseticidas e repelentes \\
R & Sistema Respiratório & 1,7 \\
V & Vários & 1,5 \\
\hline
\end{tabular}

ATC/OMS: Anatomical Therapeutic Chemical/Organização Mundial da Saúde

Fonte: os autores

\section{DISCUSSÃO}

Observou-se divergência entre as fontes de informações estudadas quanto às interações relacionadas à varfarina. Esse fator pode comprometer a assistência ofertada ao paciente, visto que a qualidade da informação sobre medicamentos deve ser considerada tão importante quanto à qualidade do princípio ativo (Marin, 2003).

O número absoluto de potenciais interações com a varfarina apresentou elevada variabilidade entre as fontes de informações (Tabela 1). Neste contexto destaca-se que existe a necessidade de analisar a relevância clínica das possíveis IM, assim como a qualidade e o nível de evidência das informações disponíveis.

Além da interação fármaco-fármaco, torna-se relevante considerar a interação fármaco-alimento e fármaco-álcool (Tabela 1), pois sabe-se que alimentos ricos em vitamina $\mathrm{K}$, por exemplo vegetais verdes folhosos, podem reduzir o efeito anticoagulante da varfarina (Greenblatt, Moltke, 2005), enquanto que a interação com álcool pode elevar o risco de hemorragia (Havrda, Mai, Chonlahan, 2005).

Além da análise da quantidade de IM, é importante destacar que a maioria das bases analisadas não classifica essas interações quanto à gravidade (Tabela 1). Dessa forma, isto pode limitar as condutas clínicas visto que algumas IM são desejadas, enquanto que outras podem comprometer a segurança e manejo do paciente. Por exemplo, os antiinflamatórios não esteroidais (AINES) apresentam elevado grau de evidência sobre riscos de interação com varfarina (Malone et al., 2004), sendo que na prática clínica esta associação pode aumentar o risco de hemorragia em 5,8 vezes (Knijff- 
Dutmer, Schut, Van De Laar, 2003). Sendo assim, além da ausência de classificação de gravidade, observa-se que nem todas as fontes de informações analisadas consideram a interação varfarina-AINES (Tabela 2).

Nas fontes de informações não se observou concordância na forma de apresentação dos fármacos, algumas fontes informam a interações baseando-se na classe farmacológica, enquanto que outras apresentam o fármaco específico (Tabela 1), esse fato pode comprometer o cuidado prestado ao paciente devido a possíveis generalizações que podem induzir a um falso-positivo ou a um falsonegativo. Exemplificando, uma das fontes consultadas apresentava interação varfarina com "Anticonvulsivos". Dessa forma, esse tipo de informação compromete a segurança do paciente, pois nesta classe de fármacos enquadram-se indutores e inibidores enzimáticos, e antiepiléticos que não provocam indução e/ou inibição das enzimas do citrocromo $\mathrm{P} 450$.

Sendo assim, os antiepilépticos indutores das isoenzimas do citocromo $\mathrm{P} 450$ (carbamazepina, fenobarbital, fenitoína e primidona) induzem o metabolismo da varfarina, reduzindo a concentração sérica, e elevando o risco de coagulação. Por outro lado, o ácido valpróico é um potente inibidor das isoenzimas do citocromo P450, aumentando a concentração sérica, e elevando o risco de hemorragia. Além disso, existem antiepilépticos de segunda e terceira geração que não exercem efeitos significativos sobre o citocromo P450 (Johannessen, Johannessen Landmark, 2010). Além dessas generalizações, destacam-se outros exemplos encontrados, tais como "Drogas para tratamento de disfunções da tireóide" e "vegetais e saladas", ambos possuindo uma gama de efeitos diferentes sobre a biodisponibilidade da varfarina.

Com relação à classificação ATC/OMS do grupo anatômico dos potenciais fármacos que interagem com a varfarina, observou-se maior prevalência de fármacos contidos no grupo do Sistema Nervoso (Tabela 3). Estudos farmacoepidemiológicos evidenciam elevação no número de prescrições de medicamentos desta classe (Queiroz-Neto, Freitas, Pereira, 2011) o que deve servir de alerta para profissionais que cuidam de pacientes que fazem uso de varfarina.

Diante do exposto verifica-se a necessidade do profissional de saúde apresentar conhecimentos clínicos e capacidade de análise crítica das diversas fontes de informações sobre IM, uma vez que a variabilidade apresentada pode comprometer o cuidado e saúde do paciente.

\section{CONCLUSÃO}

As fontes de informações apresentaram perfis heterogêneos relacionados as interações envolvendo a varfarina, destacando-se a 
discrepância entre a quantidade, a classificação e forma de apresentação das IM. Diante disso, esta variabilidade pode influenciar na qualidade do serviço de informação de medicamentos, interferindo de maneira decisiva na segurança dos pacientes. Sendo assim, torna-se imperativo que os profissionais de saúde conheçam profundamente as semelhanças e diferença entre as fontes de informações, para selecionar aquela(s) que julgar mais adequada(s) para o manejo dos pacientes.

\section{Agradecimentos}

Os autores agradecem ao Conselho Nacional de Desenvolvimento Científico e Tecnológico (CNPq) e a Coordenação de Aperfeiçoamento de Pessoal de Nível Superior (CAPES).

\section{REFERÊNCIAS}

ANSELL J, HIRSH J, POLLER L, BUSSEY H, JACOBSON A, HYLEK E. The pharmacology and management of the vitamin K antagonists: the Seventh ACCP Conference on Antithrombotic and Thrombolytic Therapy. Chest, v.126, p.204S-233S, 2004.

ANVISA - Agência Nacional de Vigilância Sanitária - Bulário eletrônico. BULA DO MAREVAN $^{\circledR}$. Disponível em < http://www4.anvisa.gov.br/base/visadoc/BM/B M\%5B25601-1-0\%5D.PDF>. Acesso em 20 de out de 2011.

BAIARDINI JR. I, GUERRA JR. L, PASQUALI M, BONADONNA P,
PASSALAQUA G, CANONICA JR. GW, et al. Quality of life in patients with adverse reactions to drugs: Preliminary results from a new questionnaire. J Allergy Clin Immunol, v.113, n.2, p.S70, 2004.

CRUCIOL-SOUZA JM, THOMSON JC. A pharmacoepidemiologic study of drug interactions in a brazilian teaching hospital.

Clinics, v.61, n.6, p.515-20, 2006

GREENBLAT DJ, MOLTKE LLV. Interaction of Warfarin With Drugs, Natural Substances, and Foods. J Clin Pharmacol, v.45, n.2, p.12732, 2005.

HAVRDA DE, MAI T, CHONLAHAN J. Enhanced antithrombotic effect of warfarin associated with low-dose alcohol consumption.

Pharmacotherapy, v.25, n.2, p.303-7, 2005.

JOHANNESSEN SI, JOHANNESSEN

LANDMARK, C. Antiepileptic Drug

Interactions - Principles and Clinical Implications. Curr Neuropharmacol, v.8, n.3, p.254-67, 2010.

KNIJFF-DUTMER EA, SCHUT GA, VAN DE LAAR MA. Concomitant coumarin-NSAID therapy and risk for bleeding. Ann

Pharmacother, v.37, n.1, p.12-16, 2003.

KONGKAEW C, NOYCE PR, ASHCROFT DM. Hospital admissions associated with adverse drug reactions: a systematic review of prospective observational studies. Ann Pharmacother. V.42, n.7, p.1017-25, 2008.

MALONE DC, ABARCA J, HANSTEN PD, GRIZZLE AJ, ARMSTRONG EP, BERGEN RCV, et al. Identification of Serious DrugDrug Interactions: Results of the Partnership to Prevent Drug-Drug Interactions. J Am Pharm Assoc,v,44, n.2, p.142-51, 2004.

MARIN, N.; LUIZA, V. L; OSORIO-DECASTRO, C. G. S.; MACHADO-DOSSANTOS, S. Assistência Farmacêutica para 
gerentes municipais de saúde. Rio de Janeiro: OPAS/OMS, 2003. 373p.

MICROMEDEX. Disponível em $<$ http://www.thomsonhc.com/hcs/librarian/ND_ T/HCS/ND_PR/Main/CS/F6892E/DUPLICAT $\mathrm{N}$

SHIELDSYNC/670910/ND_PG/PRIH/ND_B/ HCS/SBK/2/ND_P/Main/PFActionId/hcs.com mon.RetrieveDocumentCommon/DocId/2513/ ContentSetId/31/SearchTerm/warfarin\%20/Sear chOption/BeginWith\#secN13340>. Acesso em 20 de out de 2011.

OMS - ORGANIZAÇÃO MUNDIAL DE SAÚDE. WHO Model Formulary 2008. Disponível em:

$<$ http://apps.who.int/bookorders/anglais/detart1. jsp? sesslan $=1 \&$ codlan $=1 \&$ codcol $=15 \&$ codcch $=2499>$ Acesso em 20 out 2011.

OMS - ORGANIZAÇÃO MUNDIAL DE SAÚDE. ATC/DDD Index 2011. Disponível em: http://www.whocc.no/atc_ddd_index/. Acesso em 15 jun. 2011.

PATEL KJ, KEDIA MS, BAJPAI D, MEHTA SS, KSHIRSAGAR NA, GOGTAY NJ.

Evaluation of the prevalence and economic burden of adverse drug reactions presenting to the medical emergency department of a tertiary referral centre: a prospective study. BMC Clin Pharmacol. v.7, p.8, 2007.

PR VADE-MECUN ON LINE . Disponível em $<$ http://www.prvademecum.com/PRV

Ademecum Bra/PRData/NEWPrincipioActivo.asp?D=1499 \#Interacciones>. Acesso em 20 de out de 2011.
WASSERFALLEN JB, LIVIO F, BUCLIN T, TILLET L, YERSIN B, BIOLLAZ J. Rate, type, and cost of adverse drug reactions in emergency department admissions. Eur J Intern Med, v.12, n.5,p.442-47, 2001.

WINTERSTEIN AG, HATTON RC, GONZALEZ-ROTHI R, JOHNS TE, SEGAL R. Identifying Clinically Significant Preventable Adverse Drug Events Through a Database of Adverse Drug Reaction Reports. Am J Health-Syst Pharm, v.59, n.18, p.17421749, 2002.

QUEIROZ-NETO MU, FREITAS O, PEREIRA LRL. Antidepressivos e benzodiazepínicos: estudos sobre o uso racional entre usuários do SUS em Ribeirão Preto-SP. Rev Ciênc Farm Básica Apl, in press, 2011.

RANE A, LINDH JD. Pharmacogenetics of anticoagulants. Hum Genomics Proteomics, v.2010, ID:754919, 7p., 2010.

SEIZI O, BASILE AC, CARVALHO MF. Guia Zanini-Oga de Interações Medicamentosas. Atheneu Editora, 2002. 